\title{
New Final Focus System for the SLAC Linear Collider*
}

\author{
N.Toge, W.W.Ash, H.Band(a), A.O.Bazarko(b), Y.-C.Chao, R.Erickson, R.Gray, \\ S.S.Hertzbach ${ }^{(c)}$, R.R.Kofler (c), D.Mansour, C.M.Spencer, J.Turk ${ }^{(\mathrm{d})}$, C.Zeitlin ${ }^{(\mathrm{e})}$, V.Ziemann \\ Stanford Linear Accelerator Center \\ Stanford, California 94309
}

\section{Abstract}

The final focus system of the SLC has been upgraded by replacing the final quadrupole magnets with higher gradient superconducting magnets positioned closer to the interaction point. The parameters of the new system have been chosen to be compatible with the SLD detector with a minimum of changes to other final focus components. Commissioning plans for the new system are also presented.

\section{INTRODUCTION}

This paper documents the modifications made to the SLC Final Focus System (FFS) in conjunction with the replacement of the Mark II detector facility with the new SLD detector during January-May 1991.

The optical configuration of the final focus system consists of two "telescopes" pointing at each other at the IP, each of which consists of two symmetric quadrupole triplets: an upper triplet, Q4-5-6, and a high-gradient final triplet, Q12-3. For the general design philosophy of the SLC FFS system, see [1] The significant change to the beamline is the replacement of the conventional final quadrupole triplet magnets (Q1-2-3) with superconducting magnets [2].

Final Quadrupole Triplet Parameters

\begin{tabular}{|c|c|c|}
\hline & $\begin{array}{l}\text { Original } \\
\text { Configuration } \\
\text { (Mark II detector) }\end{array}$ & $\begin{array}{l}\text { New } \\
\text { Configuration } \\
\text { (SLD detector) }\end{array}$ \\
\hline Magnet conductor & Copper & $\mathrm{Cu}: \mathrm{NbTi} \quad 1.3: 1$ \\
\hline Clear aperture & $39.5 \mathrm{~mm}$ & $41 \mathrm{~mm}$ \\
\hline Triplet length & $5.3 \mathrm{~m}$ & $3.3 \mathrm{~m}$ \\
\hline Field gradient & $48.5 \mathrm{~T} / \mathrm{m}$ & $115 \mathrm{~T} / \mathrm{m}$ \\
\hline $\int \mathrm{Gdl}($ for $\mathrm{Q} 1$ ) & $460 \mathrm{kG}$ & $770 \mathrm{kG}$ \\
\hline Distance: Q1 - IP & $2.8 \mathrm{~m}$ & $2.2 \mathrm{~m}$ \\
\hline$\sigma_{\mathrm{IP}}$ & $1.5 \mu \mathrm{m}$ & $1.1 \mu \mathrm{m}$ \\
\hline$\theta_{I P}$ & $200 \mu \mathrm{rad}$ & $275 \mu \mathrm{rad}$ \\
\hline
\end{tabular}

* Supported in part by the Department of Energy, Contract DEAC03-76SF00515.

(a) University of Wisconsin, Department of Physics, Madison, Wisconsin 53706.

(b) Columbia University. Department of Physics. New York, New York 10027.

(c) University of Massachusetts, Department of Physics, Amherst, Massachusetts 01002.

(d) Yale University, Department of Physics, New Haven, Connecticut 06511.

(e) University of Oregon, Department of Physics, Eugene, Oregon 97403.

U.S. Government work not protected by U.S. Copyright.
Parameters of the original and new final triplets are listed in the table above. The field strengths listed are for $50 \mathrm{GeV}$ beams. The spot size at the interaction point $\sigma_{\mathrm{IP}}$ and the beam angular divergence $\theta_{\mathrm{IP}}$ in the table are the design values calculated assuming a normalized beam emittance of $\gamma \varepsilon=$ $3 \times 10^{-5}$ rad.m. The schematic diagram of the downstream part of the FFs beam line is shown in Figure 1.

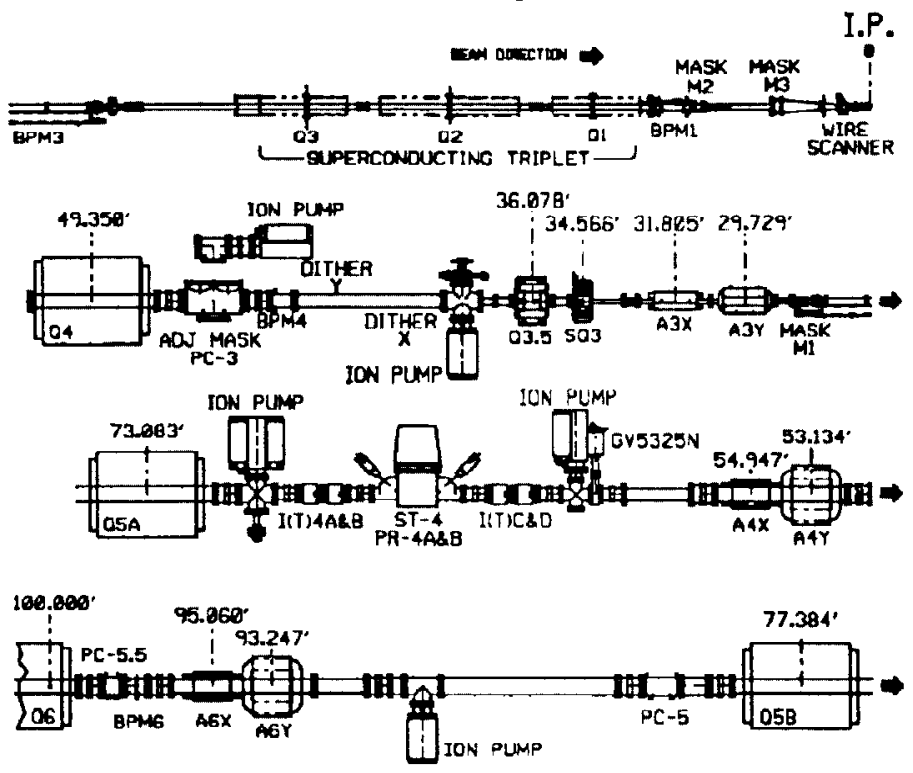

Figure 1. Schematic diagram of the downstream part of the SLC Final Focus section.

The introduction of the superconducting triplet assemblies, along with additional geometrical constraints imposed by the SLD detector design, leads to several practical consequences:

1. The final beam position monitors (BPM1 and BPM3), which are essential for beam steering across the IP and for observing beam-beam deflections, are attached to the cryostats external to the cold volume, rather than captured inside the bores of quadrupoles Q1 and Q3 as they were in the conventional configuration.

2. The three quadrupole magnets in each superconducting triplet are powered in series by a single power supply, and they have no separate trim coils. Therefore, to independently control the focal lengths (waist positions) in the horizontal and vertical planes, a new small quadrupole magnet, Q3.5, has been introduced upstream of each triplet.

3 . The relative strengths of the upstream quadrupole triplet magnets, Q4-5-6, have been changed to take advantage of 
the shorter effective focal length of the superconducting final triplets. This will yield a smaller focal spot size.

4. The presence of a small CCD vertex detector centered on the IP precludes the use of a carbon-fiber beam profile monitor [3] at that point. Instead, monitors are installed at points $21 \mathrm{~cm}$ away from the IP on each side.

This paper describes the solutions adopted to address these issues and presents the commissioning strategy being developed for this modified beamline.

\section{FINAL TRIPLET MAGNETS}

Each final triplet [4] consists of three superconducting quadrupoles [5] independently supported in a common cryostat and bath cooled by liquid helium supplied from a distant liquifier through 60-meter-long flexible low-loss transfer lines.

Strip-line beam position monitors are installed at the upstream (BPM3) and downstream ends (BPM1) of each triplet immediately outside the cryostat. Their mechanical offsets with respect to the triplet magnet center lines have been measured and incorporated into the control system database for online corrections.

The entire cryostat, together with the BPMs and primary background radiation masks, are supported by remotely operated jacks near each end, which allow horizontal and vertical position adjustments of up to $\pm 7 \mathrm{~mm}$.

\section{WAIST CONTROL, Q3.5 AND SQ3}

In the original configuration, focus adjustments were made using trim coils on the $\mathrm{Q} 2$ and $\mathrm{Q} 3$ magnets of the final quadrupole triplets. Linear combinations of these trims were used to move the longitudinal positions of the waists; i.e. the horizontal and vertical minima, to the desired collision point. The use of trim coils in the superconducting quadrupoles was rejected as unnecessarily difficult because of magnet fabrication and quench protection complications. To allow for possible variations in the relative strengths of the three quadrupoles within each triplet, tap leads were installed at the junctions between them and brought out of the cryostat through separate feed-throughs. However, a simpler method for focusing has been developed using the single triplet power supply, together with a small additional conventional quadrupole, Q3.5, located immediately upstream of the triplet. Variations in the triplet excitation will move both the $\mathrm{x}$ and $\mathrm{y}$ waists longitudinally, albeit at different rates. Q3.5 will move the $\mathrm{x}$ and $\mathrm{y}$ waists in opposite directions, and thus can be used in combination with the triplet supply to position both waists independently anywhere within about $30 \mathrm{~cm}$ of the IP. A conventional skew-quadrupole, SQ3, will be used to minimize xy crosscoupling ( $\sigma_{14}$ components of the beam at the IP).

The new Q3.5 and SQ3 quadrupoles are similar in design, requiring strengths $\int \mathrm{G} . \mathrm{dl}$ of $+/-20 \mathrm{kG}$ and $+/-10 \mathrm{kG}$, respectively, with stability and setability tolerances of $<0.05$ kG. The aperture was chosen to be 1.625 inches to allow the use of existing $0.0598^{\prime \prime}$ steel laminations which were manufactured in abundance for the original final focus magnets. With this relatively large aperture, magnetic field aberrations are negligible at the beam radius (nominally 1 $\mathrm{mm}$ ), background radiation from beam loss at this point is negligible, and vacuum conductance is good.
The magnets were assembled by stacking the laminations in a jig, welding them together, and machining the pole faces to a hyperbolic shape. A Leitz coordinate measuring machine (type PMM 12106) was used to measure each quadrant separately, and then each complete quadrupole. This was the first opportunity at SLAC to use this type of machine to examine the mechanical dimensions and shapes of quadrupole magnet poles. The coils were wound using \#6 AWG square solid copper wire, and are powered using a SLAC standard 20V bipolar power supply. These supplies have a range $+/-20$ amps and can be varied smoothly through zero. The magnetic field strengths were measured using the rotating coil method, yielding a typical sextupole content of $0.12 \%$ and a multipole sum below $0.3 \%$, measured at a radius of $1 \mathrm{~cm}$. The Q3.5 and SQ3 magnets were mounted together on a common support structure and were designed to fit in a total longitudinal space of 22.8 inches.

\section{BOOSTER POWER SUPPLY FOR Q4-5-6}

With the addition of the superconducting final triplets, the relative excitations of the upper triplet quadrupoles, Q4, Q5, and Q6, are no longer optimal. These massive quadrupoles have 5 inch apertures and are powered in scries at about 870 amps by a single large power supply. Neither their coils nor their relative beam locations could be easily modified. The solution adopted was to introduce an additional parallel power supply to boost the current through Q4 and Q6 relative to Q5 by about $13 \%$. The magnet power supply interlocks, standardization procedures, and beam optics modeling code have been updated to reflect the change.

\section{WIRE SCANNERS NEAR THE IP}

A wire profile monitor near the IP has been critically important in the development of the SLC, especially at times following modifications to the final focus hardware, when the relative $x / y$ offsets of the two opposing beams have to be known accurately to establish collisions. In principle, the BPMs can determine the relative beam positions at the IP within a few $\mu \mathrm{m}$; however, their electrical offsets are different for electrons and positrons, leading to significant systematic errors for this purpose.

Due to the presence of a new precision CCD vertex detector at the IP, a wire monitor could not be installed exactly at the IP. Instead, two sets of wire monitors have been installed such that wires may be inserted at points $21 \mathrm{~cm}$ from the IP on either side. In principle, the beams can be brought into collision at either of these positions by shifting the relative bunch timing by $+/$ - one damping ring rf bucket. The carbon fibers ( $32 \mu \mathrm{m}$ and $4 \mu \mathrm{m}$ diameter) are mounted on an aluminum fork which can be inserted into the path of the beam with a mechanism driven by three piezoelectric "inch-worms". The beams can be swept across the wires using small air-core steering dipoles. Bremsstrahlung radiation created by a beam hitting a wire is detected with a Cerenkov detector located downstream along the beam line. By correlating the bremsstrahlung signal with the excitation of the stcering magnets, the beam position relative to the wire can be deduced with an accuracy of a few microns. 


\section{COMMISSIONING STRATEGY}

The first goal in the commissioning work will be to establish beam collisions with the new superconducting triplets. This "cold-start" will be done as follows :

1. The beam lines up to stoppers ST4 (between Q4 and Q5) on both sides of the IP have remained essentially unchanged. The incoming electron beam will first be transported through the north arc and parked on the north ST4 stopper while the beam line optical properties up to this point are optimized and the radiation level in the collider hall is checked.

2. ST4 north will then be retracted, allowing the electron beam to cross the IP to stopper ST4 south (on the outgoing side). With this arrangement, polarity and functional tests can be performed on the BPMs and steering correction dipole magnets in the IP area.

3. The alignment of the two triplets and their associated BPMs can then be checked with respect to each other and with the nominal beam line. This is done by observing the beam trajectory while varying the excitation of the triplet magnets from zero field to the full nominal field. The mechanical misalignments of the triplets and the electrical offsets of the BPMs can then be calculated and compensated using the support jacks and software parameters, respectively.

4. ST4 south will then be pulled out, and the electron beam will be transported through the south final focus system to where it is extracted to the main dump.

5. The procedure above is then repeated using the positron beam, starting from the south side.

6. The electron beam spot will be focused at a point $21 \mathrm{~cm}$ from the IP using one of the carbon fiber profile monitors as a guide. This can then be repeated at the corresponding point on the other side of the IP. By interpolating between the resulting focusing parameters, the horizontal and vertical waists can be translated to the nominal IP position. This process will be repeated using the positron beam. The resulting spot sizes at the IP are expected to be on the order of a few microns, and the transverse distance between the beams is expected to be on the order of $100 \mu \mathrm{m}$.

7. If necessary, the profile wires can be used to guide steering to bring the beams into collision. Beam-beam deflection signals become clearly observable when the beams are within $100 \mu \mathrm{m}$ of each other if the spot sizes are less than about $10 \mu \mathrm{m}$ and the bunch intensities are greater than about $10^{10}$ particles per pulse. The beam-beam deflection signals can then be used to guide the focusing and steering adjustments to arbitrarily fine precision.

\section{CONCLUSIONS}

The SLC Final Focus system has been modified with the installation of superconducting final triplets, and the final assembly and alignment are nearing completion. The recommissioning program is scheduled to commence in midMay 1991.

\section{ACKNOWLEDGEMENTS}

We wish to express our gratitude to the SLAC engineering and technical staff whose efforts made this work possible; critical contributions have been made by W.Burges, M.Nordby, J.Cobb, R.Gaxiola, M.Pietryka, T.King, M.Berndt, W. Michaels, K. Johnson, T.Porter, W.Pierce and A.Grillo.

\section{REFERENCES}

[1] J.J. Murray, K.L. Brown, T. Fieguth, The Completed Design of the SLC Final Focus System, SLAC-PUB-4219, February 1987, and Proceedings of the 1987 IEEE Particle Accelerator Conference, Washington, D.C., March 16-19, 1987, p. 1331.

[2] R. Erickson, T. Fieguth, and J. J. Murray, Superconducting Quadrupoles for the SLC Final Focus, SLAC-PUB-4199 (January 1987), and Proceedings of the 1987 IEEE Particle Accelerator Conference, Washington, D.C., March 16-19. 1987 , p. 142.

[3] C. Field, N. Hower, and B. Scott, Nucl. Inst. Methods A295 (1990), p.279.

[4] W.W. Ash et al. Superconducting Final Focus for the SLAC Linear Collider, to be published.

[5] A.D. McInturff, J. A. Carson, H. E. Fist, R. A. Erickson, The Magnetic Properties of the SLC Intersection Region Superconducting Quadrupole Triplets. SLAC-PUB-4478, November 1987, and Tenth International Conference on Magnet Technology, Boston, MA, September 23-26, 1987. 